\title{
Overview of the Fourth Flight of the ANITA Experiment
}

\section{Jiwoo Nam* for the ANITA Collaboration}

LeCosPA and Dept. of Phys., National Taiwan University

E-mail: jwnamephys.ntu.edu.tw

The ANITA (ANtarctic Impulsive Transient Antenna) is a NASA long-duration balloon payload with an array of radio antennas. The primary goal of ANITA is to search for ultra-high energy cosmogenic neutrinos by detecting their impulsive radio signals emitted via the Askaryan effect in the Antarctic ice sheet. Moreover, ANITA is able to detect radio impulses produced from ultrahigh energy cosmic-ray showers as result of their emissions via interaction with the geomagnetic field. The ANITA-IV experiment was launched on December 2, 2016, and successfully completed 28 days of flight. We will present the ANITA-IV instrument, flight operation, calibration as well as the status of the data analysis. 
The ANITA (ANtarctic Impulsive Transient Antenna, shown in Figure 1) experiment is a NASA balloon-borne neutrino telescope, aiming to detect astrophysical neutrinos with energies above $10^{18} \mathrm{eV}$. In such energies, the presence of neutrinos is predicted to be almost certain by the Greisen, Zatsepin and Kuzmin (GZK) effect $[1,2]$, which is the $p+\gamma$ interaction, occurred when hadronic UHECRs propagate through intergalactic space which is filled with the $3 \mathrm{~K}$ cosmic microwave background radiation. The UHECR data obtained from many ground based experiments $[3,4,5,6]$ provide plausible evidence supporting the existence of the GZK cut-off which would be ultimately confirmed through the absolute flux measurements of the UHE neutrinos.

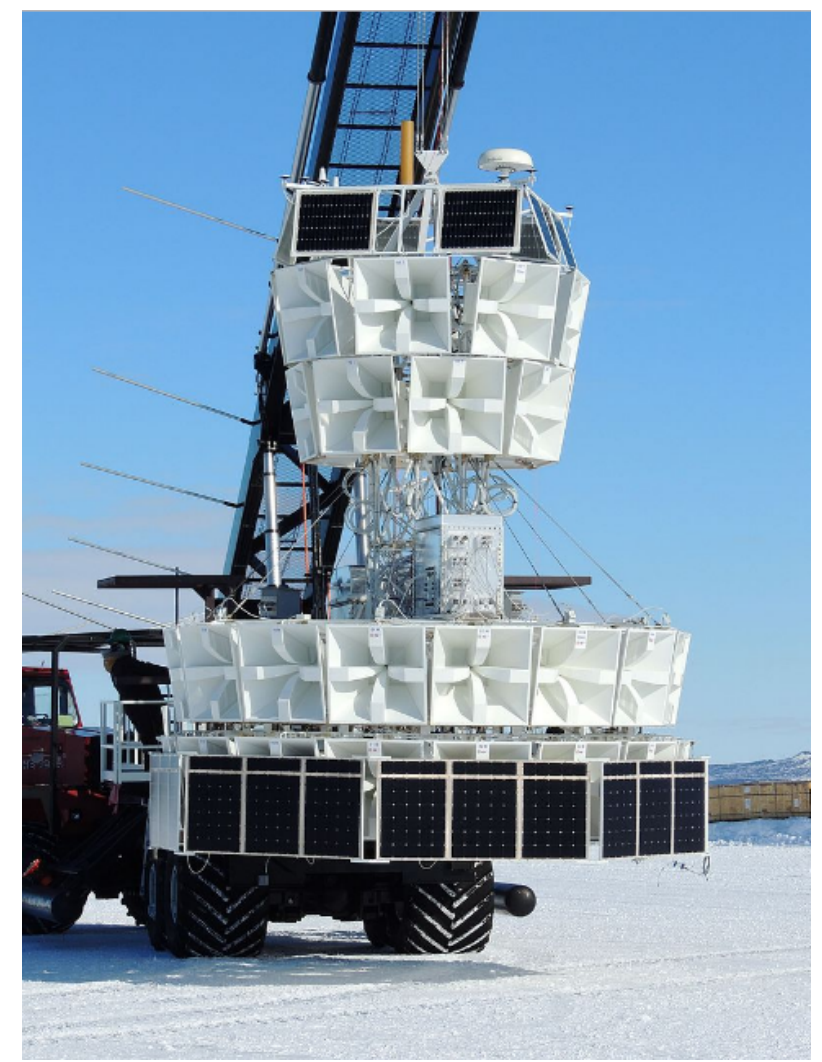

Figure 1: The ANITA-4 payload under preparation for launch, credit Luke Batten.

Detection of UHE neutrinos is experimentally challenging because of their small cross-section and extremely low rates in the energy range which requires very large target volumes. Even the largest neutrino detector, such as IceCube with an effective volume of $\sim 1 \mathrm{~km}^{3}$, would require a decade of operation time to detect handful numbers of events GZK neutrinos.

ANITA's approach is unique in the method of obtaining the effective volume required for UHE neutrino detection. Floating in the Antarctic stratosphere, ANITA looks down on the ice sheet to detect the radio signals generated along the development of showers which are induced by the neutrino interaction with the Antarctic ice. From 30-40 km above the sea level which is the typical altitude of NASA long duration balloon flight, the horizon distance is about $700 \mathrm{~km}$, which makes uses of the vast volume of thick ice $(\sim 2 \mathrm{~km})$ within the horizon as the target.

It was suggested by Askaryan in the 1960s that a shower could generate a strong radio signal 
during development in dielectric media such as Antarctic ice, and that this could be applied to detection of UHE particles [7, 8]. The Askaryan effect has been confirmed experimentally using various media in the 2000s. The system response of ANITA was well characterized by using real Askaryan signals from an ice target [9]. Thanks to the Askaryan effect, together with the radio-transparent characteristic in cold Antarctic ice with long attenuation lengths in the order of a kilometer, the quietest radio environment in the planet, as well as advanced radio technology and long-range balloon technology, we have established ANITA as the most sensitive experiment for UHE neutrino detection in energies $E>10^{19} \mathrm{eV}$ [10].

In addition to the UHE neutrinos, as the primary subject of the project, ANITA is also sensitive to UHE cosmic rays [11]. The production mechanism of the radio signals from UHE cosmic rays is independent of the Askaryan effect, which is a synchrotron-like process; the relativistic electrons and positions in extensive air shower (EAS) produce coherent emission as a result of interaction with the geomagnetic field. Since the geomagnetic field in Antarctica is almost vertical, the electron positons are horizontally separated by the Lorentz force, resulting in horizontally polarized radio pulse. Although ANITA's altitude is much higher than the shower maximum, the down going signal can be detected by reflection on the ice surface with high radio reflectivity; the surface of the Antarctic is used as a large radio reflector. The vertical component of the polarization is further suppressed through the reflection due to low reflectivity for s-polarized waves. On the other hand, the Askaryan signal from the neutrino interaction is affected in the opposite way; when the radio signal produced in the ice passes through the ice-air interface, the p-polarization is suppressed: therefore the vertical polarization remains as the predominant signal. This orthogonality of the polarizations is an important characteristic used to distinguish between neutrino and cosmic ray signals. ANITA has detected of order 20 UHE cosmic ray events, with a mean energy $3 \times 10^{18} \mathrm{eV}$, including 3 direct events that arrived almost horizontally without reflection. In addition, the ANITA team confirmed this geomagnetic emission in the laboratory, the T-510 experiment [12], which led to a better understanding of this radiation, allowing simulation to improve systematic uncertainties. Analysis techniques for UHE cosmic rays have also been greatly improved, especially in the energy measurement [13]. Based on spectral slope analysis of the RF signal, the off-axis angle from the shower axis was determined, which allowed the $20-50 \%$ of uncertainty in energy measurement.

Recently, ANITA has reported an unexpected event, in which the arrival direction of the radio pulse was the upward direction like the other reflected cosmic ray events, but the polarity was opposit, as a signature of non-reflection[14]. This event can be interpreted as an upcoming airshower, but its origin remains unknown. It may be thought of as a tau decay signal from Earth skimming tau neutrino interaction, but the Earth penetration length corresponding to the arrival elevation angle is too long to be explained by the neutrino cross section by the standard model. A more detailed characterization of this event will be discussed at another presentation in this conference[15].

ANITA has completed a total of four flights since 2002; ANITA-1 (35 days, December 2002January 2003), ANITA-2 (30 days, December 2008-January 2009), ANITA-3 (22 days, December 2014-January 2015), and ANITA-4 (28 days, December 2016). Rather than simply repeating previous experiments to accumulate time, each flight has increased the sensitivity by using improved instruments. ANITA-4 was basically the same instrument as ANITA-3, which is a wide band $(180-1200 \mathrm{MHz})$ antenna array consisted of 48 dual-polarized quad-ridged horn antennas with 
$10 \mathrm{dBi}$ gain, arranged an azimuthally symmetric array of 2 cluster groups of $8+8$ upper, 16 middle, and 16 lower antennas with a downward cant of $10^{\circ}$ to cover the horizon down to $40^{\circ}$ of the nadir. There were several significant changes and improvements in the RF front-end, the RF filters, in the trigger system.

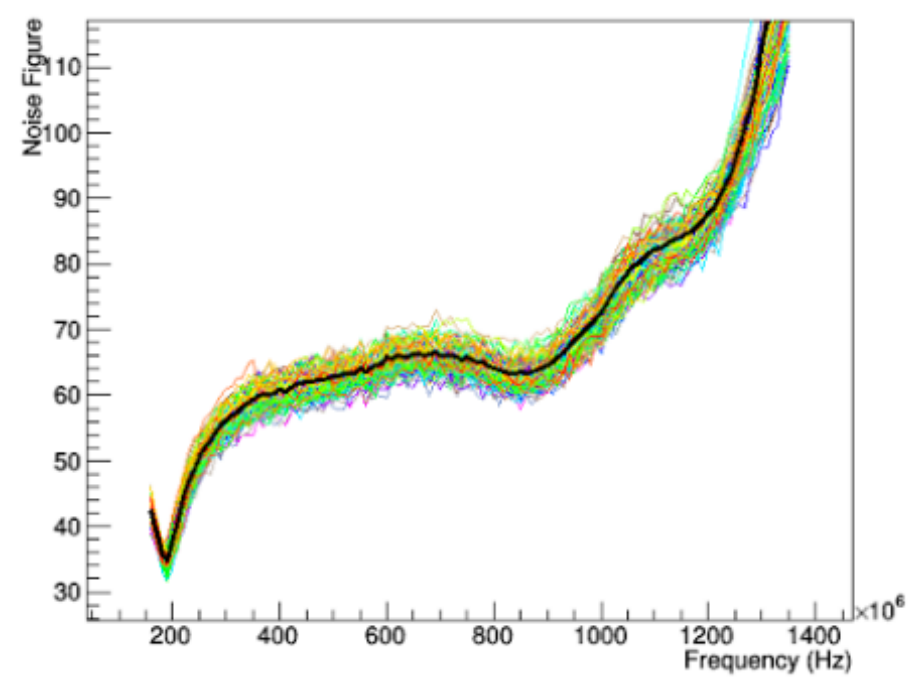

Figure 2: The measured noise temperature of all 96 RF front-end units for ANITA-4 flight.

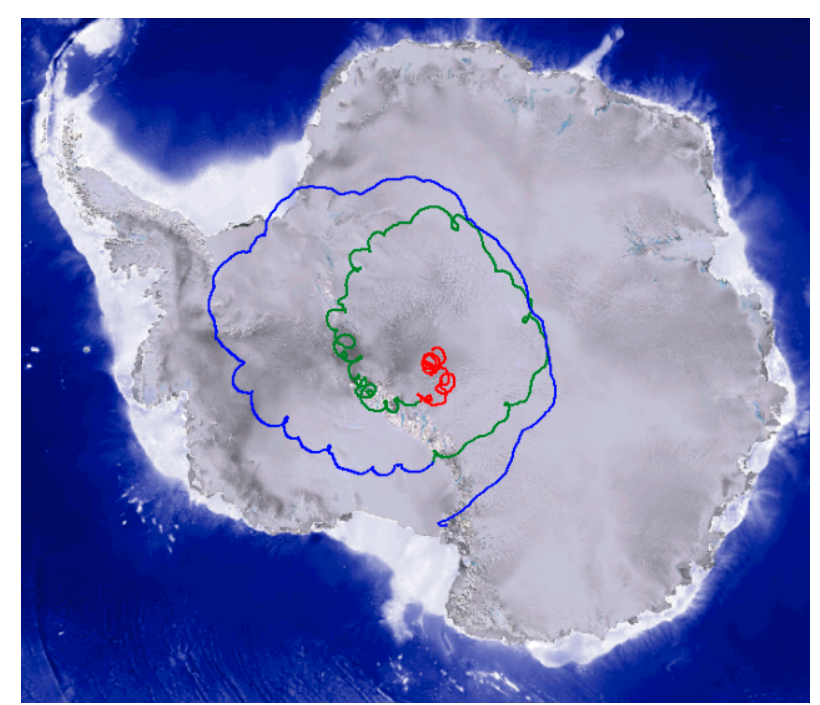

Figure 3: ANITA-4 flight trajectory.

The RF front-end consists of a front-end filter, followed by a low-noise-amplifier (LNA), a high- and low-pass filter combination, a second stage booster amplifier, and an equalizer. Prior flights used bandpass filters in the front-end, but in ANITA-4, we employed only low pass filters to reduce the transmission loss, noting the fact that the antenna cut-off was stiff enough below 180 MHz. In ANITA-4, we used customized LNAs which were the most utilized for the ANITA frequency band in all channels, compared to ANITA-3, which were only used on 1/3 channels. 
Figure 2 shows the measured noise temperature of the RF front-end of all 96 channels in ANITA4, which is $30-40 \mathrm{~K}$ improvement compared to ANITA-3, resulting in a $20 \%$ energy threshold reduction.

One of the problems encountered during ANITA-3 flight was an unexpected electromagnetic interference, mainly continuous waveform (CW) noise at $260 \mathrm{MHz}$ and $360 \mathrm{MHz}$, which were produced by newly launched satellites. Since this noise appears only in the direction in which the satellite exists, the effect of the noise can be minimized by masking the corresponding channels from the trigger. However, this substantially reduced overall exposure at a level of $40 \%$. In order to solve this problem, ANITA-4 built and employed the Tunable Universal Filter Frontend (TUFF) unit with three independent tunable- and switchable- notch filters in each RF channel. Because of narrow notch widths $(\sim 50 \mathrm{MHz})$, the effect of the signal loss and the noise increase by the filter is small compared to preventing the exposure loss caused by the CW noise.

A new feature of the ANITA-4 trigger is that the radio signals need to be linearly polarized. Taking advantage of the Askaryan and the geomagnetic radiation signals being linearly polarized, which suppresses circularly polarized noise such as the electromagnetic interferences from satellites. This was implemented by converting the Horizontal- and Vertical-polarization signals to Left- and Right-Polarization through a 90 degree hybrid coupler and then requiring a coincidence of them at the Level-1 trigger. Further trigger conditions in the higher levels are for temporal coincidence of multiple antennas located in adjacent $\phi$-sectors, which are basically the same as the prior flights [16].

The ANITA-4 payload was launched from Williams Field on December 2, 2016 at 13:10 UTC, and landed at $88^{\circ} 13.51 \mathrm{~S}, 98^{\circ} 52.99 \mathrm{E}$ (125 nm away from the South Pole) on December 30, 2016 after 28 days at float. As seen in Figure 3, the trajectory of ANITA-4 had a spiral pattern centered on the South pole, which resulted in ANITA moving away from the McMurdo Station area, where the anthropogenic noise level is highest in the Antarctica. In addition to the ANITA payload, we launched two follow-on pulser payloads, called HiCal [17], for a study of radio reflection characteristics on the ice surface and validation and calibration of ANITA system. ANITA-4 was partially recovered 10 days after the landing, and all important but small instruments, such as data disks and amplifiers, were safely recovered. Full recovery of ANITA-4 is scheduled in next season 2017.

ANITA-4 was in pristine condition for most of the flight time and recorded about 100 million events. It maintained $94 \%$ livetime on average, achieved a total 27.3 days of the live time. As described above, Using TUFF, ANITA-4 successfully minimized the expose loss due to electromagnetic interference from satellites, which resulted in an improvement of 3.6 times effective exposure compared to ANITA-3.

Impulsive radio pulses generated from two ground pulser systems were used for in-flight system validation and calibration which include characterization of the trigger response, system gains, time offsets, antenna coordinates, and determination of the pointing resolution. Figure 4 shows pointing resolution determined by using the Western Antarctic Ice Seet (WAIS) Divide pulser data. Here, the directions of the received radio pulses were obtained by the interferometry technique which is the kernel of ANITA data analysis [18], and the results show excellent pointing capability of ANITA-4; angular resolutions for vertical and horizontal-directions are $\sigma_{\theta}=0.15^{\circ}$ and $\sigma_{\phi}=0.5^{\circ}$, respectively.

Figure 5 shows a couple of preliminary candidate events detected by ANITA-4; one predom- 

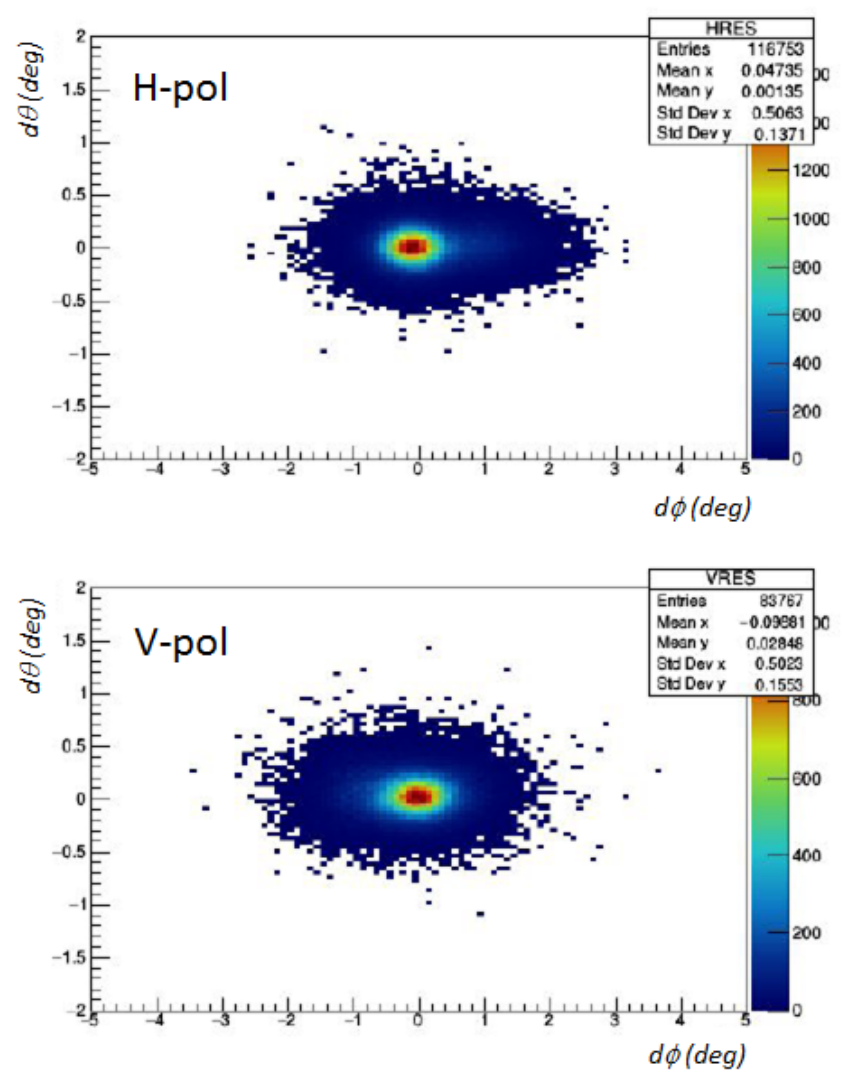

Figure 4: Pointing resolution of ANITA-4: Angular difference between reconstructed and predicted angle. Top figure is for H-pol and bottom for V-pol.

inates in the horizontal polarization (the top figure) and the other in the vertical polarization (the bottom figure). These events were found in the on-line telemetry data during the flight operation and monitoring shifts when ANITA was flying in deep field area. The identification of these events is left to be done in detailed data analysis followed. The ANITA-4 data analysis is currently in progress and is in parallel with ANITA-3 data analysis which is close to completion.

Through ANITA flights in the past one and half decades, we have made steady improvements in the data analysis, such as the energy estimation of cosmic ray events as described above, digital filtering methods especially for $\mathrm{CW}$ noise. Various advanced methods, such as the template search technique that finds signal events having expected waveforms, and the binned approach that optimizes event selections reflecting the RF noise environment according to event locations, are beining investgated.

Combining all data of the prior flights, ANITA-4 would provide the strongest probe to exam the neutrino physics in energies above $10^{19} \mathrm{eV}$. The total UHE cosmic rays sample in the ANITA-3 data could be of order of a hundred or more, which in itself is an excellent science topic, but is also a precious calibration sample determining the sensitivity of ANITA instrument. The interesting up-coming shower event which may be interpreted as tau neutrino, would also detect whether it is reproduced in ANITA-4 data. Beside of the UHE neutrinos and cosmic rays, many science topics, 


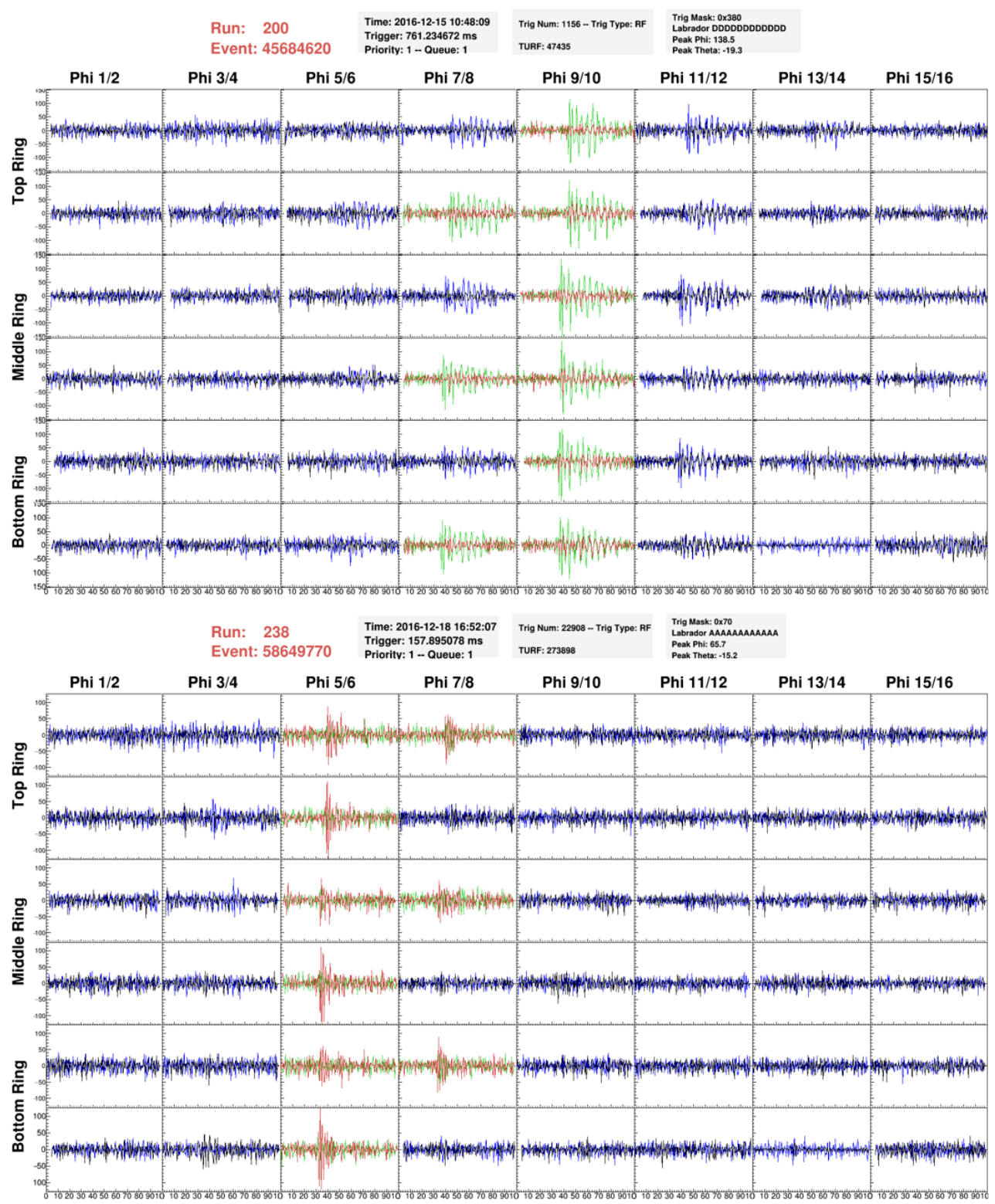

Figure 5: Examples of impulsive events.

such as a search for GRB neutrinos [19], searches for exotic particles such as monopole and dark matter, and also recently a search for fast radio bursts, have been parts of the ANITA data analysis

We are thankful for support from the National Aeronautics and Space Administration, the National Science Foundation Office of Polar Programs, the Department of Energy Office of Science High Energy Physics Division, the UK Science and Technology Facilities Council, and the Taiwan Ministry of Science and Technology. A special debt of gratitude is owed to the staff of the Columbia Scientific Balloon Facility. 


\section{References}

[1] K. Greisen, Phys. Rev Lett. 16,748 (1966)

[2] G.T. Zatsepin and V. A. Kuzmin, JETP Letters 4, 78 (1966)

[3] D.R. Bergman et al., Nucl. Phys. Proc. Suppl.165:19-26 (2007)

[4] J. Abraham et al., Phys. Rev. Lett. 101, 061101 (2008)

[5] J. Abraham et al., Phys. Lett. B685, 239-246 (2010)

[6] P. Tinyakov et al., Nucl. Instr. Meth. A 742, 29-34 (2014)

[7] G. A. Askaryan, JETP 14, 441 (1962)

[8] G. A. Askaryan, JETP 21, 658.(1965)

[9] P. Gorham et al., Phy. Rev. Lett. 99, 171101 (2007)

[10] P. Gorham et al., Phy. Rev. Lett. 103, 051103 (2009)

[11] S. Hoover et al., Phys. Rev. Lett. 105, 151101 (2010)

[12] K. Belov et al. Phys. Rev. Lett. 116, 141103 (2016)

[13] H. Schoorlemmer et al., Astroparticle Physics, 77, 32-43 (2016)

[14] P.W. Gorham, et al., Phys. Rev. Lett. 117, 071101 (2016)

[15] A. Romero-Wolf, et al., presentation at ICRC 2017 (2017)

[16] P. Gorham et al., Astroparticle Physics, 32, 10-41 (2010)

[17] P. W. Gorham et al., J. Astron. Instrum. 06, 1740002 (2017)

[18] A. Romero-Wolf et al., Astroparticle Physics 60, 72-85 (2015)

[19] A. G. Vieregg et al., Astro. Phys. J. 736, No 1 (2011)

[20] M. Detrixhe et al. Phys. Rev. D 83, 023513 (2011) 